\title{
Catalytic Decarboxylative sp-sp3 Coupling
}

\section{Supporting Information: Table of Contents.}

General procedure for catalytic hydroarylation. $\quad$ S-1

Spectral characterization of 2a $\quad$ S-2

Spectral characterization of $\mathbf{2 c} \quad$ S-3

Spectral characterization of $\mathbf{2 d} \quad$ S-4

Spectral characterization of $2 \mathrm{e} \quad$ S-5

Spectral characterization of $\mathbf{2 f} \quad$ S-6

Spectral characterization of $\mathbf{2 g} \quad$ S-7

Spectral characterization of $\mathbf{2 h} \quad$ S-8

Spectral characterization of $2 \mathbf{i} \quad$ S-9

Spectral characterization of $\mathbf{2} \mathbf{j} \quad S-10$

Spectral characterization of $\mathbf{2 m} \quad$ S-11

Spectral characterization of $\mathbf{2 n} \quad$ S-12

Spectral characterization of $20 \quad$ S-13

Spectral characterization of $\mathbf{2 p} \quad$ S-14

Spectral characterization of $\mathbf{2 q} \quad$ S-15

Spectral characterization of trans-2s $\quad$ S-16

Spectral characterization of $\mathbf{3 r} \quad$ S-17

Crossover experiment $-{ }^{1} \mathrm{H}$ NMR spectra $\quad$ S-18-19

General Procedure for the Palladium-Catalyzed Decarboxylation reaction: A roundbottom side-arm flask $(25 \mathrm{~mL})$ containing $\mathrm{Pd}\left(\mathrm{PPh}_{3}\right)_{4}(0.050 \mathrm{mmol}, 10.0 \mathrm{~mol} \%)$ was evacuated and purged with argon gas. Allylic alkynoate $1(0.50 \mathrm{mmol})$ and dry, degassed toluene $(3.0 \mathrm{~mL})$ were added to the flask and the reaction mixture was stirred at $75{ }^{\circ} \mathrm{C}$ for 2-48 h. Then the reaction mixture was cooled to room temperature, diluted with dichloromethane. The mixture was filtered through a short Celite and silica gel pad and washed with dichloromethane several times. The filtrate was concentrated and the residue was purified on a silica gel column using hexane-ethyl acetate (95:5) as eluent to afford the 1,4-enyne products. 
2a:<smiles>C(#Cc1ccccc1)/C=C/c1ccccc1</smiles>
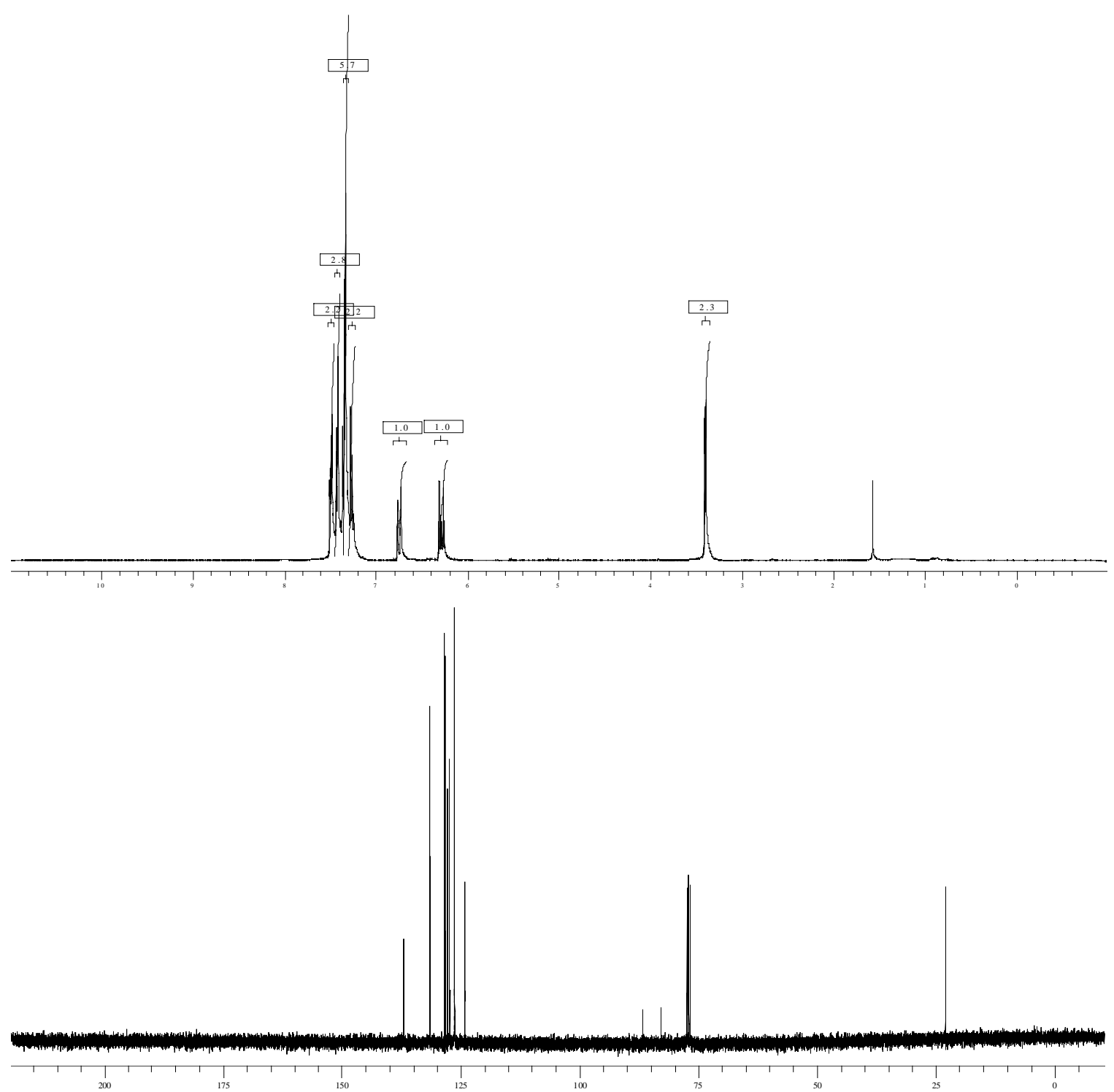

(E)-Pent-1-en-4-yne-1,5-diyldibenzene (2a): ${ }^{1} \mathrm{H}$ NMR (400 MHz, $\left.\mathrm{CDCl}_{3}\right): \delta=3.39$ $(\mathrm{dd}, J=1.7 \mathrm{~Hz}, J=5.5 \mathrm{~Hz}, 2 \mathrm{H}), 6.27(\mathrm{dt}, J=5.5 \mathrm{~Hz}, J=15.7 \mathrm{~Hz}, 1 \mathrm{H}), 6.74(\mathrm{dt}, J=1.7$ $\mathrm{Hz}, J=15.7 \mathrm{~Hz}, 1 \mathrm{H}), 7.25(\mathrm{t}, J=7.3 \mathrm{~Hz}, 1 \mathrm{H}), 7.30-7.36(\mathrm{~m}, 6 \mathrm{H}), 7.41(\mathrm{~d}, J=7.6 \mathrm{~Hz}, 2$ $\mathrm{H}), 7.47(\mathrm{t}, J=7.3 \mathrm{~Hz}, 2 \mathrm{H}) ;{ }^{13} \mathrm{C}\left\{{ }^{1} \mathrm{H}\right\} \mathrm{NMR}\left(100 \mathrm{MHz}, \mathrm{CDCl}_{3}\right): \delta=22.9,82.8,86.6$, 123.5, 124.2, 126.2, 127.3, 127.8, 128.2, 128.5, 131.3, 131.5, 137.0; IR (neat): 964, 1070, 1267, 1342, 1488, 1596, 2918, 3029, $3056 \mathrm{~cm}^{-1}$; HRMS calcd for (M+) $\mathrm{C}_{17} \mathrm{H}_{14} 218.1096$, found 218.1096. 
2c:
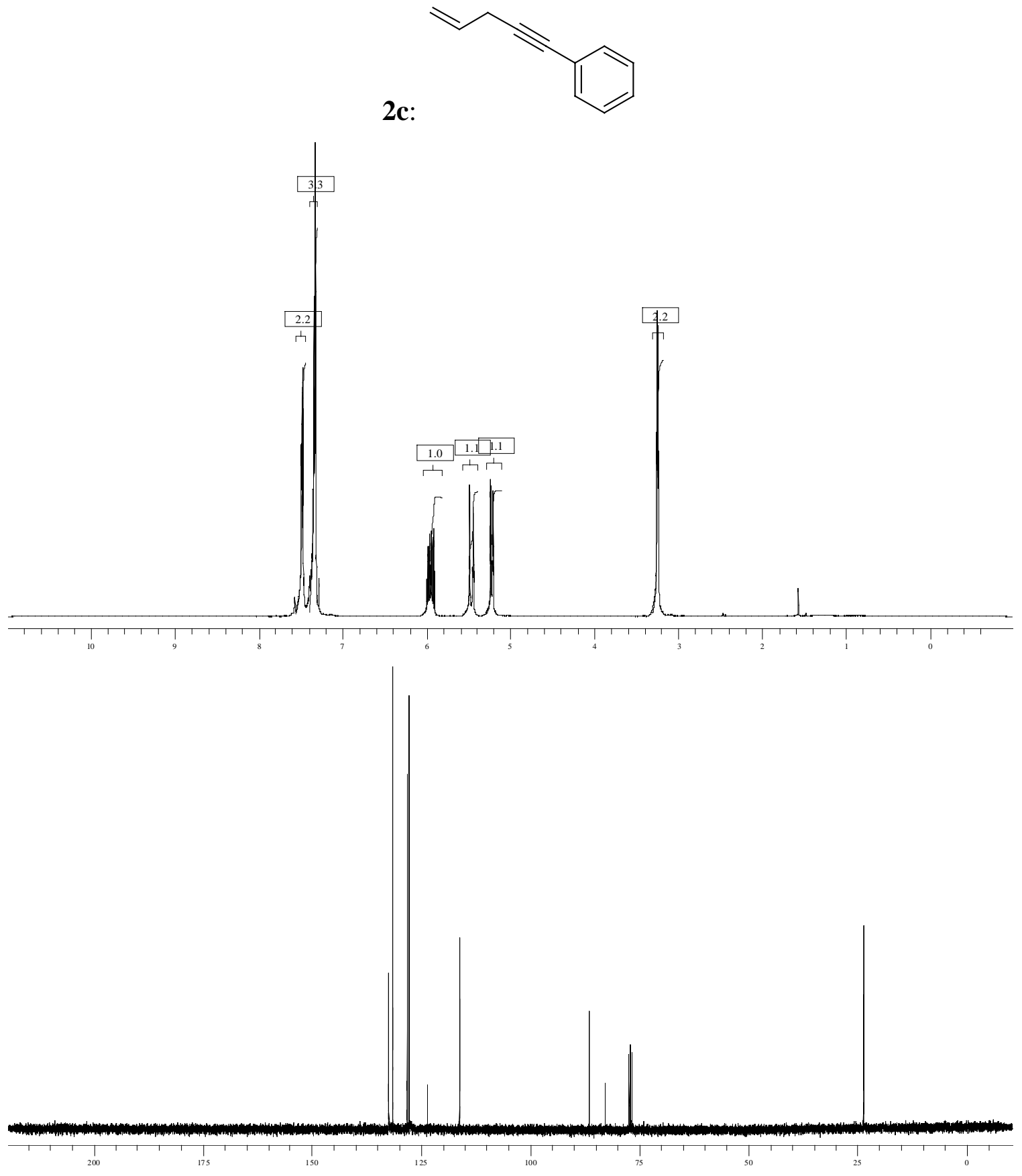

Pent-4-en-1-ynylbenzene (2c): ${ }^{\text {lb }}{ }^{1} \mathrm{H}$ NMR $\left(400 \mathrm{MHz}, \mathrm{CDCl}_{3}\right): \delta=3.22(\mathrm{~d}, J=5.2 \mathrm{~Hz}, 2$ H), $5.19(\mathrm{dt}, \mathrm{J}=1.7 \mathrm{~Hz}, J=9.9 \mathrm{~Hz}, 1 \mathrm{H}), 5.43(\mathrm{dt}, J=1.7 \mathrm{~Hz}, J=16.4 \mathrm{~Hz}, 1 \mathrm{H}), 5.88$ 5.97 (m, $1 \mathrm{H}), 7.29-7.33(\mathrm{~m}, 3 \mathrm{H}), 7.43-7.46$ (m, $2 \mathrm{H}) ;{ }^{13} \mathrm{C}\left\{{ }^{1} \mathrm{H}\right\}$ NMR (100 MHz, $\left.\mathrm{CDCl}_{3}\right)$ : $\delta=23.6 .82 .8,86.5,116.2,127.7,128.1,128.4,131.5,132.4$; IR (neat): 690, 756, 916, 989, 1070, 1442, 1488, 1641, 2916, 3031, $3058 \mathrm{~cm}^{-1}$; HRMS calcd for $(\mathrm{M}+) \mathrm{C}_{11} \mathrm{H}_{10}$ 142.0783, found $\mathrm{C}_{11} \mathrm{H}_{10} 142.0783$. 


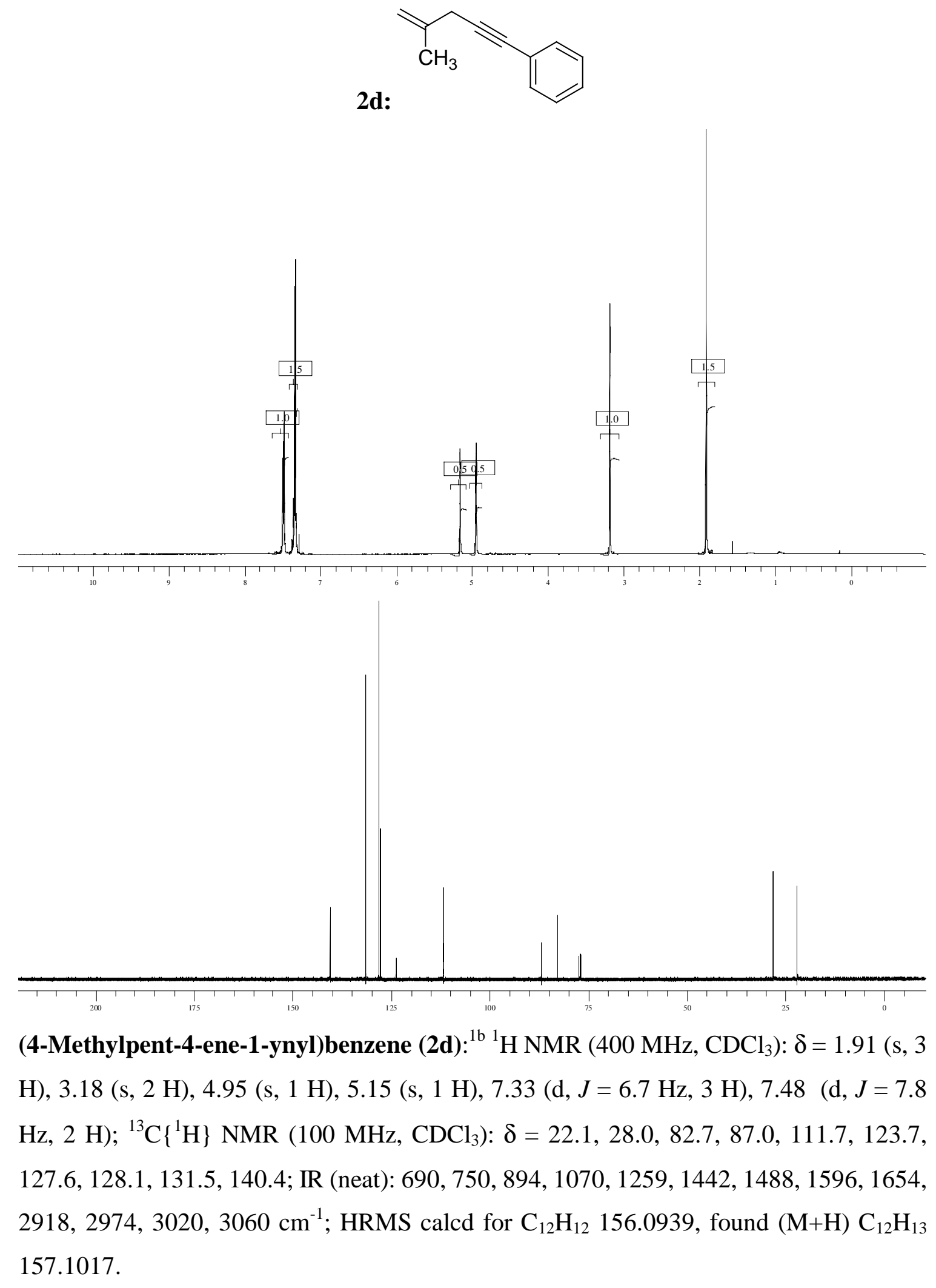



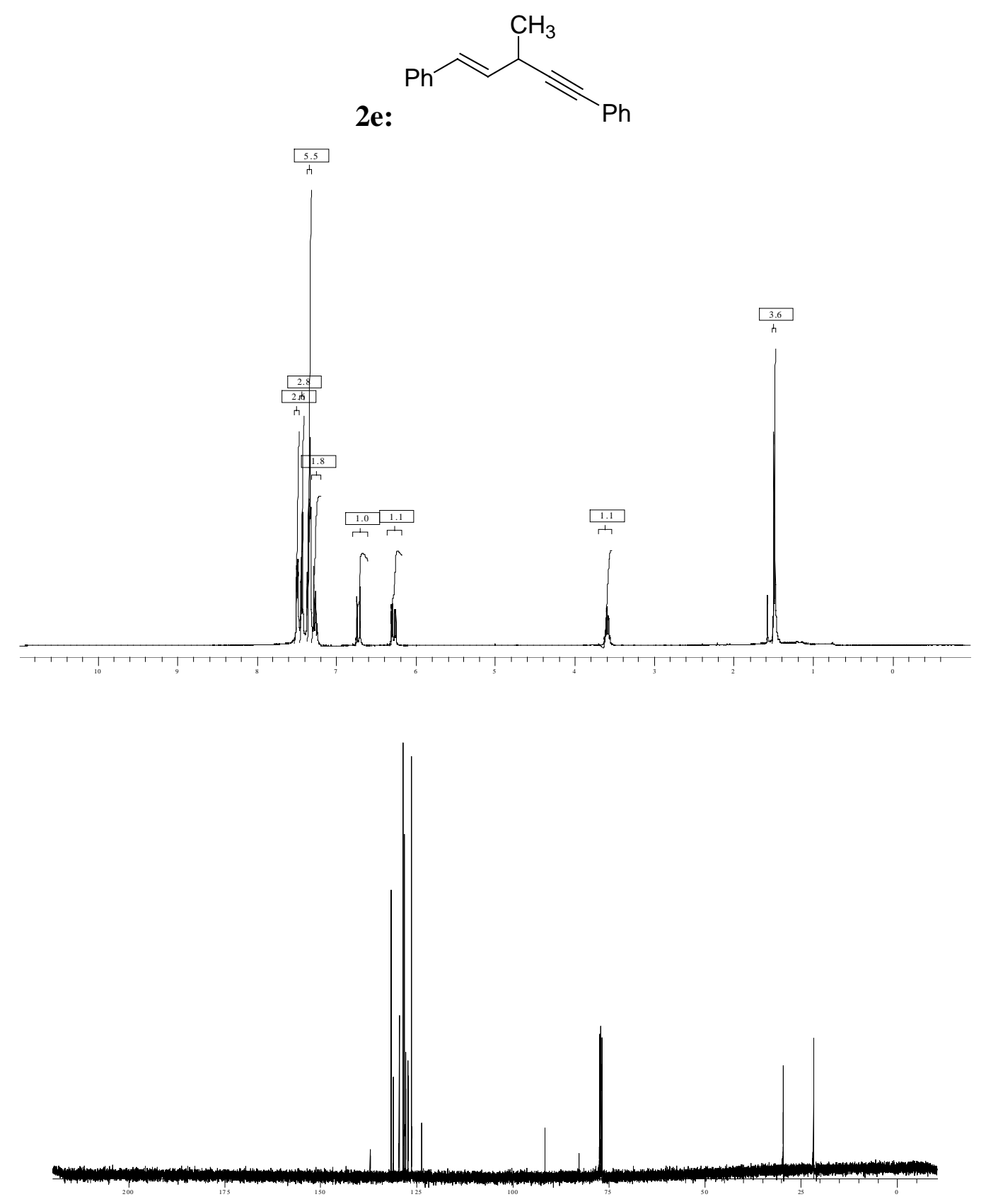

(E)-(3-methylpent-1-en-4-yne-1,5-diyl)dibenzene (2e): ${ }^{2} \mathrm{H}$ NMR (400 $\left.\mathrm{MHz}, \mathrm{CDCl}_{3}\right)$ :

$\delta=1.48(\mathrm{~d}, J=7.0 \mathrm{~Hz}, 3 \mathrm{H}), 3.55-3.62(\mathrm{~m}, 1 \mathrm{H}), 6.28(\mathrm{dd}, J=6.1 \mathrm{~Hz}, J=15.7 \mathrm{~Hz}, 1 \mathrm{H})$, $6.72(\mathrm{~d}, J=15.7 \mathrm{~Hz}, 1 \mathrm{H}), 7.26(\mathrm{~d}, \mathrm{~J}=7.6 \mathrm{~Hz}, 1 \mathrm{H}), 7.32-7.37$ (m, $5 \mathrm{H}), 7.43$ (d, $J=7.8$ $\mathrm{Hz}, 2 \mathrm{H}), 7.49$ (d, $J=7.6 \mathrm{~Hz}, 2 \mathrm{H}) ;{ }^{13} \mathrm{C}\left\{{ }^{1} \mathrm{H}\right\} \mathrm{NMR}\left(100 \mathrm{MHz}, \mathrm{CDCl}_{3}\right): \delta=21.7,29.5$, 82.6, 91.6, 123.6, 126.3, 127.3, 127.7, 128.1, 128.5, 129.4, 131.0, 131.6, 137.0; IR (neat): 690, 760, 892, 966, 983, 1442, 1488, 1596, 1654, 2921, 2974, 3020, $3060 \mathrm{~cm}^{-1}$; HRMS calcd for $(\mathrm{M}+) \mathrm{C}_{18} \mathrm{H}_{16}$ 232.1252, found $\mathrm{C}_{18} \mathrm{H}_{16} 232.1125$. 
2f:
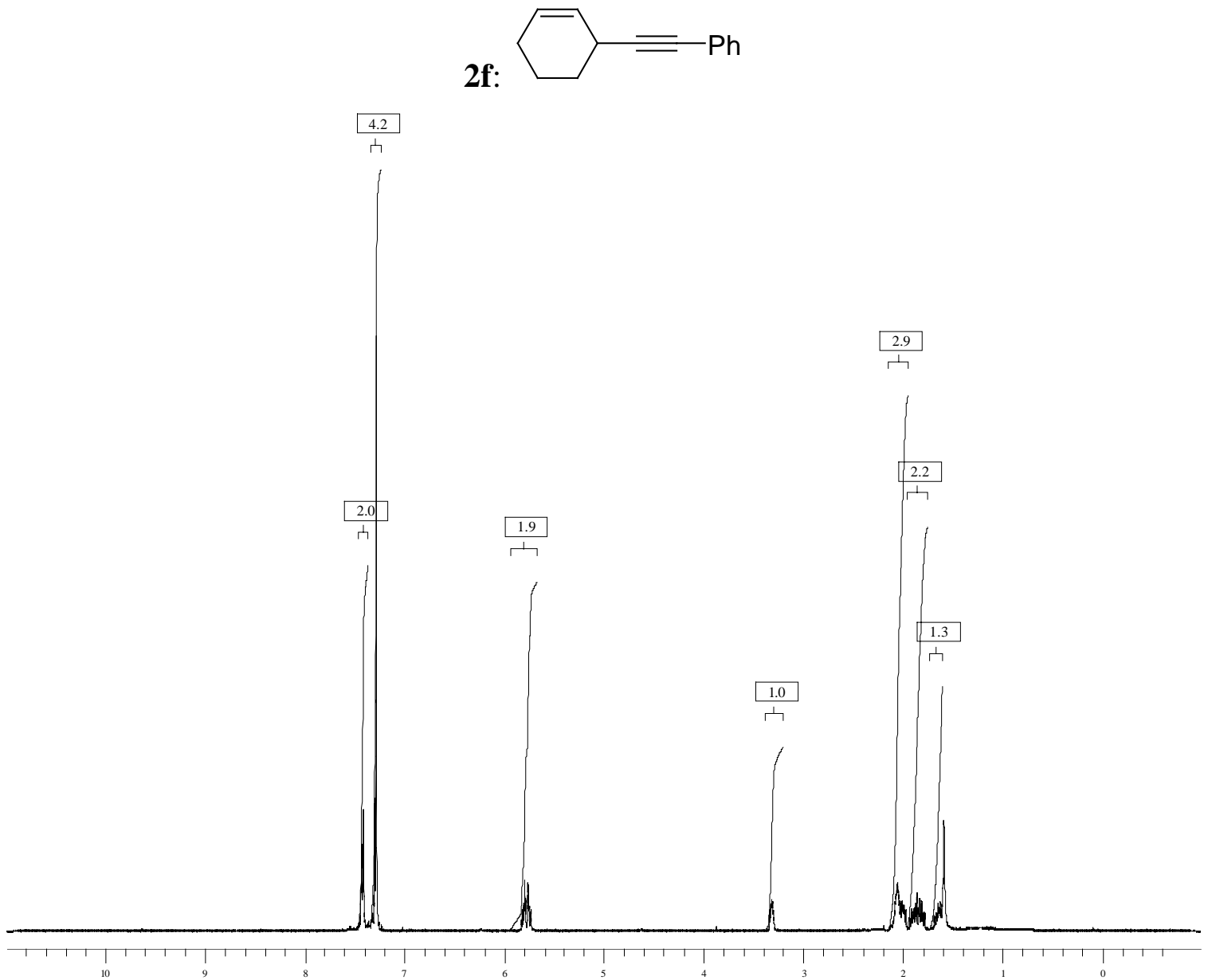

(Cyclohex-2-enylethynyl)benzene (2f): ${ }^{1 \mathrm{~b}, 2}{ }^{1} \mathrm{H}$ NMR (400 MHz, $\mathrm{CDCl}_{3}$ ): $\delta=1.61-1.68$ (m, $1 \mathrm{H}), 1.78-1.93$ (m, $2 \mathrm{H}), 1.97-2.09$ (m, $3 \mathrm{H}), 3.29-3.33$ (m, $1 \mathrm{H})$, 5.72-5.78 (m, $2 \mathrm{H})$, $7.29(\mathrm{t}, J=6.8 \mathrm{~Hz}, 3 \mathrm{H}), 7.41(\mathrm{~d}, J=7.0 \mathrm{~Hz}, 2 \mathrm{H}) ;{ }^{13} \mathrm{C}\left\{{ }^{1} \mathrm{H}\right\} \mathrm{NMR}\left(100 \mathrm{MHz}, \mathrm{CDCl}_{3}\right): \delta$ $=20.6,24.6,27.9,29.3,80.2,92.8,123.8,127.0,127.5,128.0,128.1,131.5$; IR (neat): 692, 754, 893, 964, 1172, 1443 1488, 1643, 2838, 28.61, 2927, $3031 \mathrm{~cm}^{-1}$. 

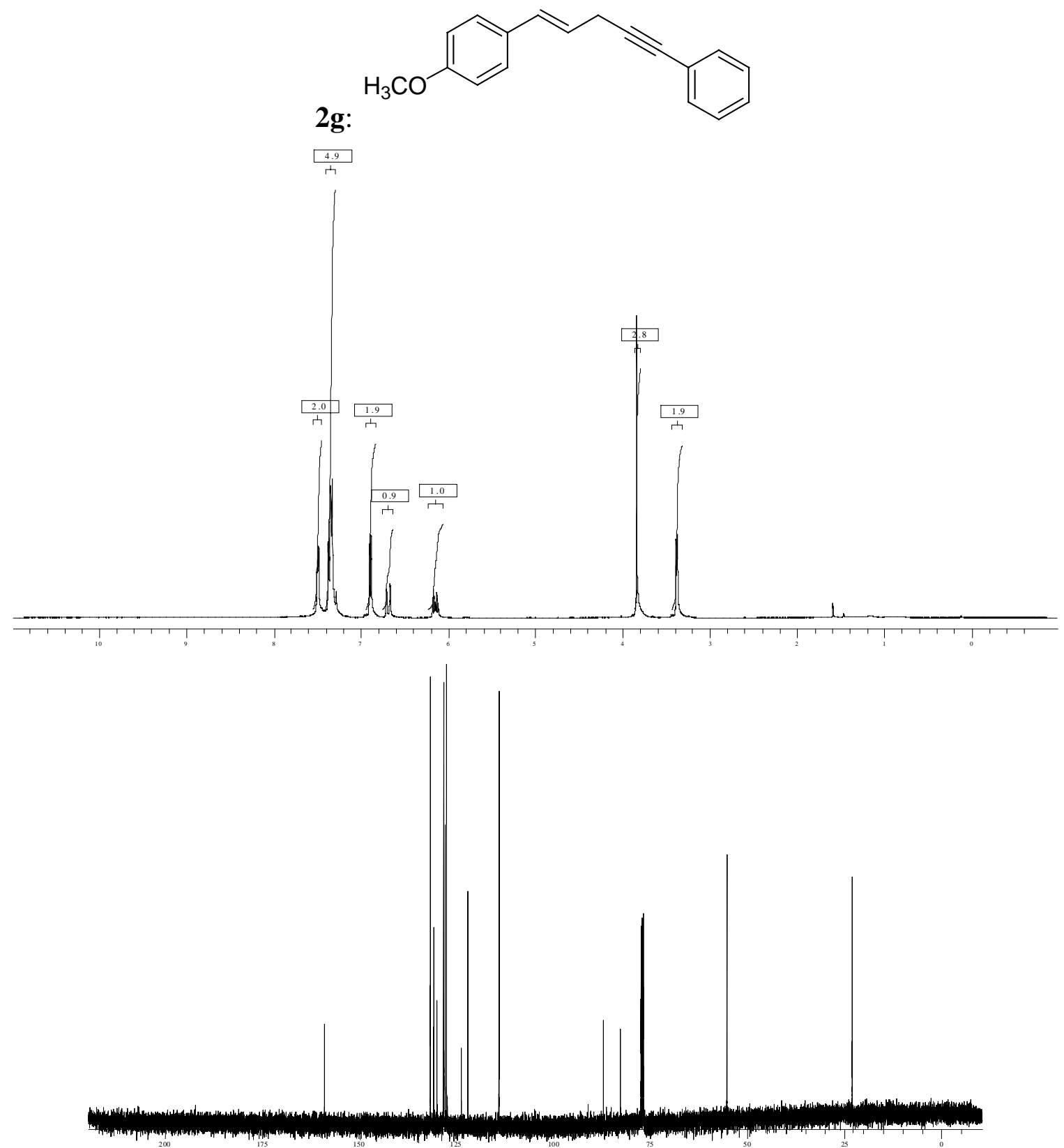

(E)-1-Methoxy-4-(5-phenylpent-1-en-4-ynyl)benzene $\quad(\mathbf{2 g}):{ }^{1} \mathrm{H} \quad \mathrm{NMR}(400 \mathrm{MHz}$, $\left.\mathrm{CDCl}_{3}\right): \delta=3.38(\mathrm{~d}, J=5.5 \mathrm{~Hz}, 2 \mathrm{H}), 3.84(\mathrm{~s}, 3 \mathrm{H}), 6.14(\mathrm{td}, J=5.55 \mathrm{~Hz}, J=15.7 \mathrm{~Hz}, 1$ H), $6.68(\mathrm{~d}, J=15.7 \mathrm{~Hz}, 1 \mathrm{H}), 6.88(\mathrm{dd}, J=6.7 \mathrm{~Hz}, J=1.7 \mathrm{~Hz}, 2 \mathrm{H}), 7.32-7.37(\mathrm{~m}, 5 \mathrm{H})$, 7.48-7.49 (m, $2 \mathrm{H}) ;{ }^{13} \mathrm{C}\left\{{ }^{1} \mathrm{H}\right\} \mathrm{NMR}\left(100 \mathrm{MHz}, \mathrm{CDCl}_{3}\right): \delta=22.9,55.3,82.6,87.0,113.8$, 121.9, 123.6, 127.3, 127.7, 128.2, 129.8, 130.7, 131.5, 158.9; IR (neat): 757, 1029, 1174, 1249, 1512, 1600, 1720, 2929, 3002, $3060 \mathrm{~cm}^{-1}$; HRMS calcd for $(\mathrm{M}+) \mathrm{C}_{18} \mathrm{H}_{16} \mathrm{O}$ 248.1201, found (M-H) $\mathrm{C}_{18} \mathrm{H}_{15} \mathrm{O} 247.1123$. 


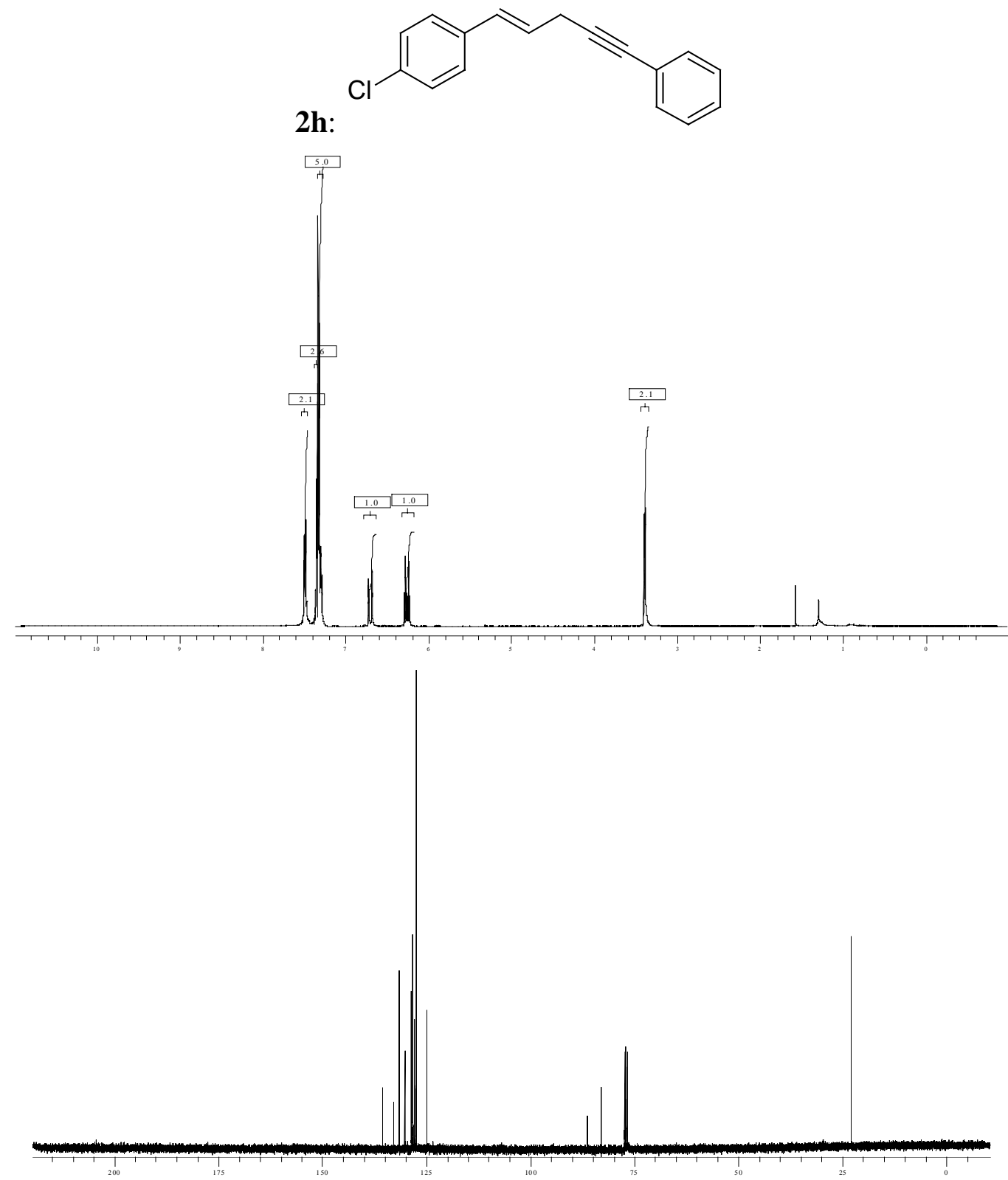

(E)-1-Chloro-4-(5-phenylpent-1-en-4-ynyl)benzene (2h): ${ }^{1} \mathrm{H}$ NMR (400 MHz, $\left.\mathrm{CDCl}_{3}\right)$ : $\delta=3.39(\mathrm{dd}, J=2.0 \mathrm{~Hz}, J=5.5 \mathrm{~Hz}, 2 \mathrm{H}), 6.26(\mathrm{dt}, J=5.5 \mathrm{~Hz}, J=15.8 \mathrm{~Hz}, 1 \mathrm{H}), 6.70(\mathrm{dt}$ $, J=2.0 \mathrm{~Hz}, J=15.7 \mathrm{~Hz}, 1 \mathrm{H}), 7.28-7.33(\mathrm{~m}, 5 \mathrm{H}), 7.34-7.36(\mathrm{~m}, 2 \mathrm{H}), 7.49(\mathrm{dd}, J=2.3$ $\mathrm{Hz}, \mathrm{J}=6.1 \mathrm{~Hz}, 2 \mathrm{H}) ;{ }^{13} \mathrm{C}\left\{{ }^{1} \mathrm{H}\right\} \mathrm{NMR}\left(100 \mathrm{MHz}, \mathrm{CDCl}_{3}\right): \delta=22.9,83.0,86.3,124.9$, 127.4, 127.8, 128.2, 128.3, 128.6, 130.1, 131.6, 132.8, 135.5; IR (neat): 690, 756, 1014, 1091, 1267, 1490, 1595, 1699, 2850, 2921, $3060 \mathrm{~cm}^{-1}$; HRMS calcd for $(\mathrm{M}+) \mathrm{C}_{17} \mathrm{H}_{13} \mathrm{Cl}$ 252.0706, found $\mathrm{C}_{17} \mathrm{H}_{13} \mathrm{Cl} 252.0706$. 


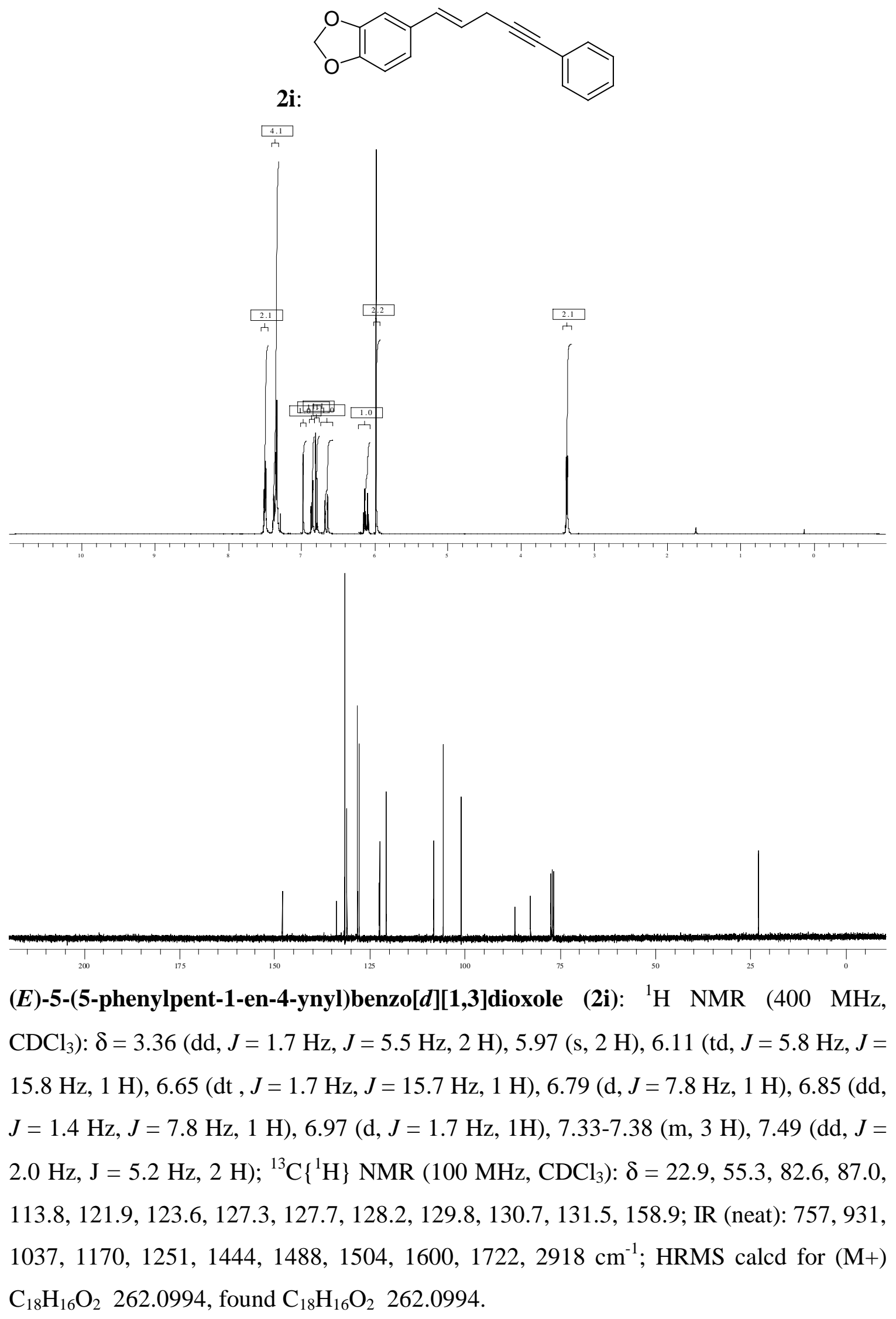



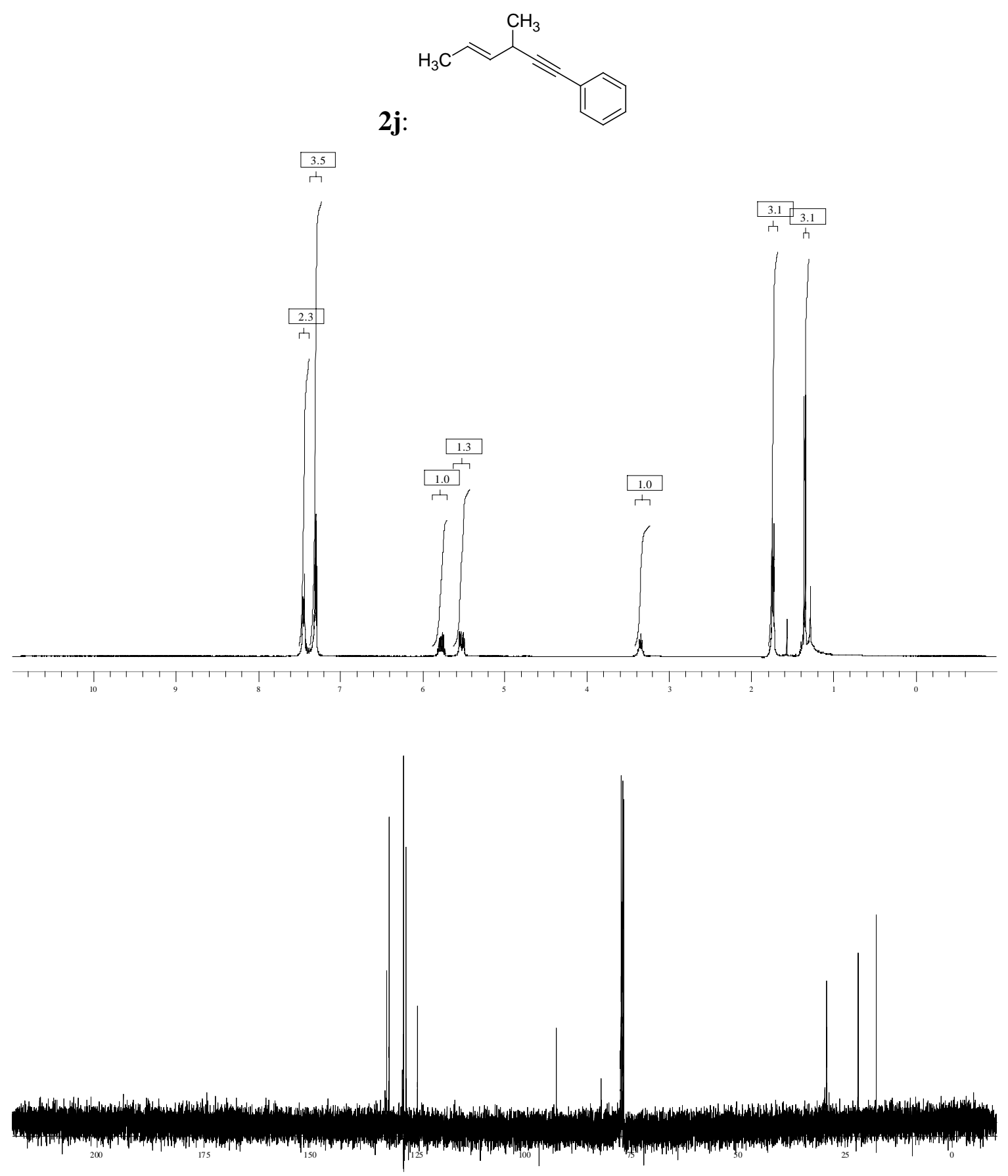

(E)-(3-methylhex-4-en-1-ynyl)benzene (2j): ${ }^{2} \mathrm{H}$ NMR (400 MHz, $\left.\mathrm{CDCl}_{3}\right): \delta=1.34$ (d, $J=7.0 \mathrm{~Hz}, 3 \mathrm{H}), 1.73(\mathrm{td}, J=1.4 \mathrm{~Hz}, J=6.4 \mathrm{~Hz}, 3 \mathrm{H}), 3.31-3.38(\mathrm{~m}, 1 \mathrm{H}), 5.48-5.55(\mathrm{~m}$, $1 \mathrm{H})$, 5.72-5.81 (m, $1 \mathrm{H}), 7.29-7.32(\mathrm{~m}, 3 \mathrm{H}), 7.43-7.45(\mathrm{~m}, 2 \mathrm{H}) ;{ }^{13} \mathrm{C}\left\{{ }^{1} \mathrm{H}\right\}$ NMR $(100$ $\left.\mathrm{MHz}_{\mathrm{CDCl}}\right): \delta=17.6,21.8,29.2,81.9,92.4,124.8,127.5,128.1,131.5,132.0$; IR (neat): 754, 964, 1056, 1242, 1488, 1643, 1757, $2981 \mathrm{~cm}^{-1}$; HRMS calcd for $(\mathrm{M}+) \mathrm{C}_{13} \mathrm{H}_{14}$ 170.1096, found $\mathrm{C}_{13} \mathrm{H}_{14} 170.1111$. 

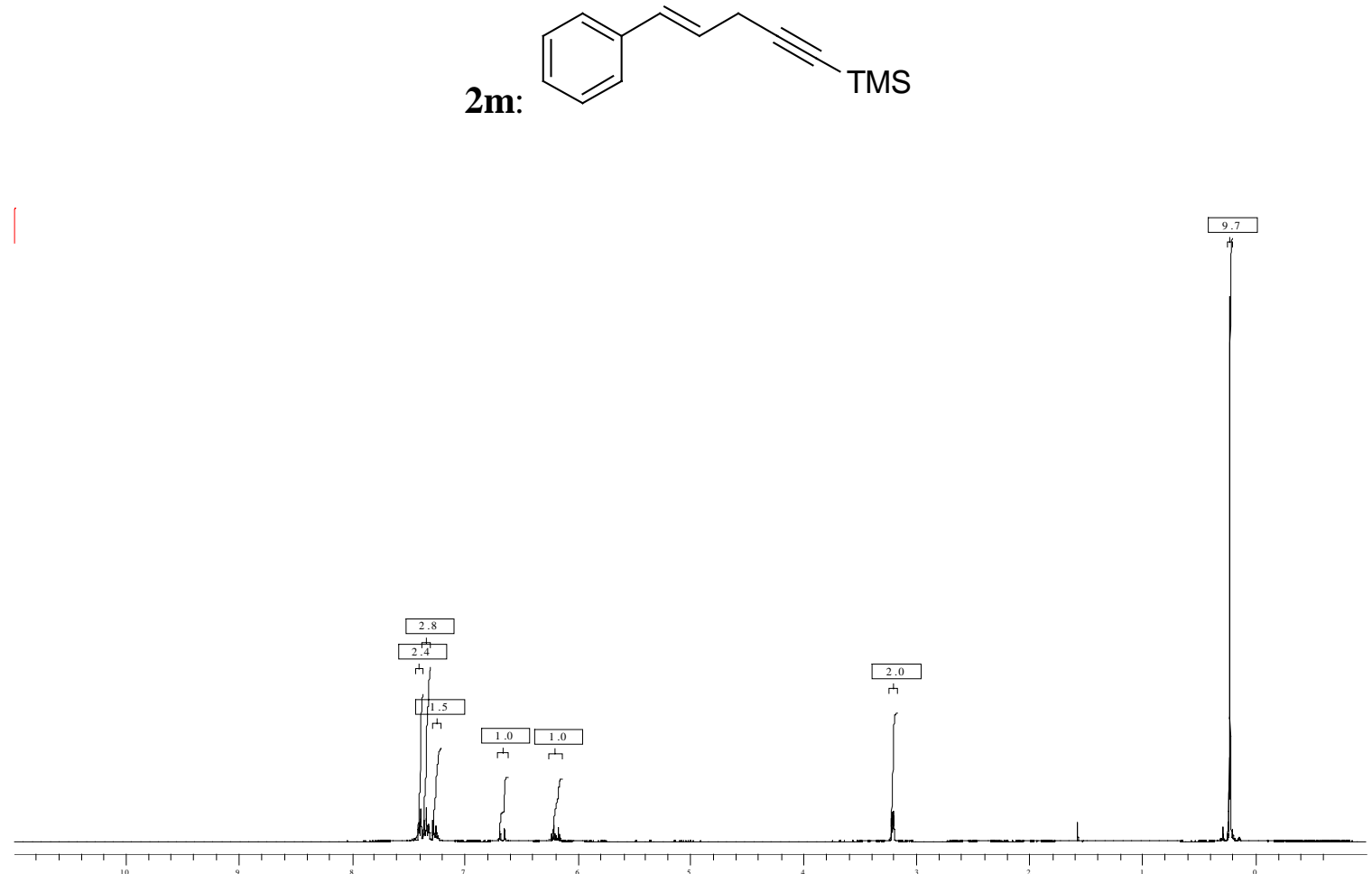

(E)-Trimethyl(5-phenylpent-4-en-1-ynyl)silane (2m): ${ }^{1} \mathrm{H}$ NMR (400 $\left.\mathrm{MHz}, \mathrm{CDCl}_{3}\right): \delta$ $=0.22(\mathrm{~s}, 9 \mathrm{H}), 3.20(\mathrm{dd}, J=2.0 \mathrm{~Hz}, J=5.8 \mathrm{~Hz}, 2 \mathrm{H}), 6.18(\mathrm{dt}, J=2.0 \mathrm{~Hz}, J=5.8 \mathrm{~Hz}, 1$ H), $6.66(\mathrm{~d}, J=15.7 \mathrm{~Hz}, 1 \mathrm{H}), 7.26$ (d, J=7.6 Hz, $1 \mathrm{H}), 7.34$ (t, $J=7.3 \mathrm{~Hz}, 2 \mathrm{H}), 7.38$ (d, $J=7.8 \mathrm{~Hz}, 2 \mathrm{H}) ;{ }^{13} \mathrm{C}\left\{{ }^{1} \mathrm{H}\right\} \mathrm{NMR}\left(100 \mathrm{MHz}, \mathrm{CDCl}_{3}\right): \delta=0.1,23.4,87.0,103.6,123.9$, 126.2, 127.3, 128.5, 131.4, 137.0; IR (neat): 692, 759, 843, 964, 1027, 1172, 1249, 1488, 1643, 1735, 2927, 2950, $3031 \mathrm{~cm}^{-1}$; HRMS calcd for $\mathrm{C}_{14} \mathrm{H}_{18} \mathrm{Si} 214.1178$, found (M+) $\mathrm{C}_{14} \mathrm{H}_{18} \mathrm{Si} 214.1178$. 
<smiles>CC#CCC=Cc1ccc2c(c1)OCO2</smiles>
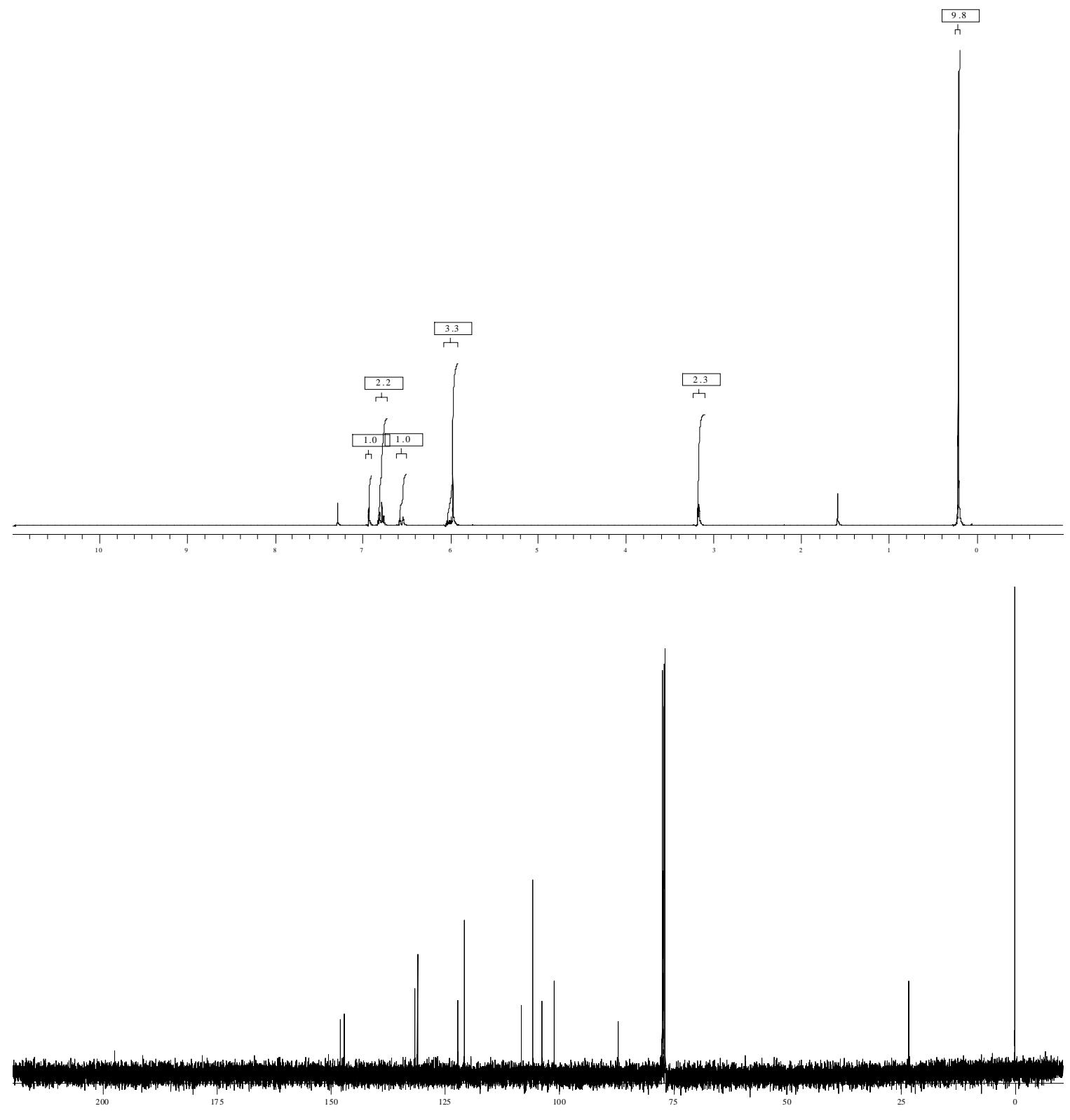

1-[(E)-5-Phenyl-1-penten-4-ynyl]benzene (2n): ${ }^{1} \mathrm{H}$ NMR (400 $\left.\mathrm{MHz}, \mathrm{CDCl}_{3}\right): \delta=0.22$ $(\mathrm{s}, 9 \mathrm{H}), 3.17(\mathrm{dd}, J=2.0 \mathrm{~Hz}, J=5.8 \mathrm{~Hz}, 2 \mathrm{H}), 5.96(\mathrm{~s}, 2 \mathrm{H}), 6.00(\mathrm{dt}, J=5.5 \mathrm{~Hz}, J=$ $15.4 \mathrm{~Hz}, 1 \mathrm{H}), 6.56(\mathrm{dt}, J=1.7 \mathrm{~Hz}, J=15.7 \mathrm{~Hz}, 1 \mathrm{H}), 6.56(\mathrm{~d}, J=7.8 \mathrm{~Hz}, 1 \mathrm{H}), 6.81(\mathrm{dd}$, $J=1.4 \mathrm{~Hz}, J=7.8 \mathrm{~Hz}, 1 \mathrm{H}), 6.90(\mathrm{~d}, J=1.4 \mathrm{~Hz}, 1 \mathrm{H}) ;{ }^{13} \mathrm{C}\left\{{ }^{1} \mathrm{H}\right\} \mathrm{NMR}(100 \mathrm{MHz}$, $\left.\mathrm{CDCl}_{3}\right): \delta=0.0,23.3,86.9,100.9,103.7,105.6,108.2,120.7,122.2,130.9,131.5,146.9$, 147.9; IR (neat): 759, 844, 935, 1039, 1191, 1246, 1446, 1490, 1504, 2896, $2958 \mathrm{~cm}^{-1}$; HRMS calcd for $(\mathrm{M}+) \mathrm{C}_{15} \mathrm{H}_{18} \mathrm{O}_{2} \mathrm{Si} 258.1076$, found 258.1076. 

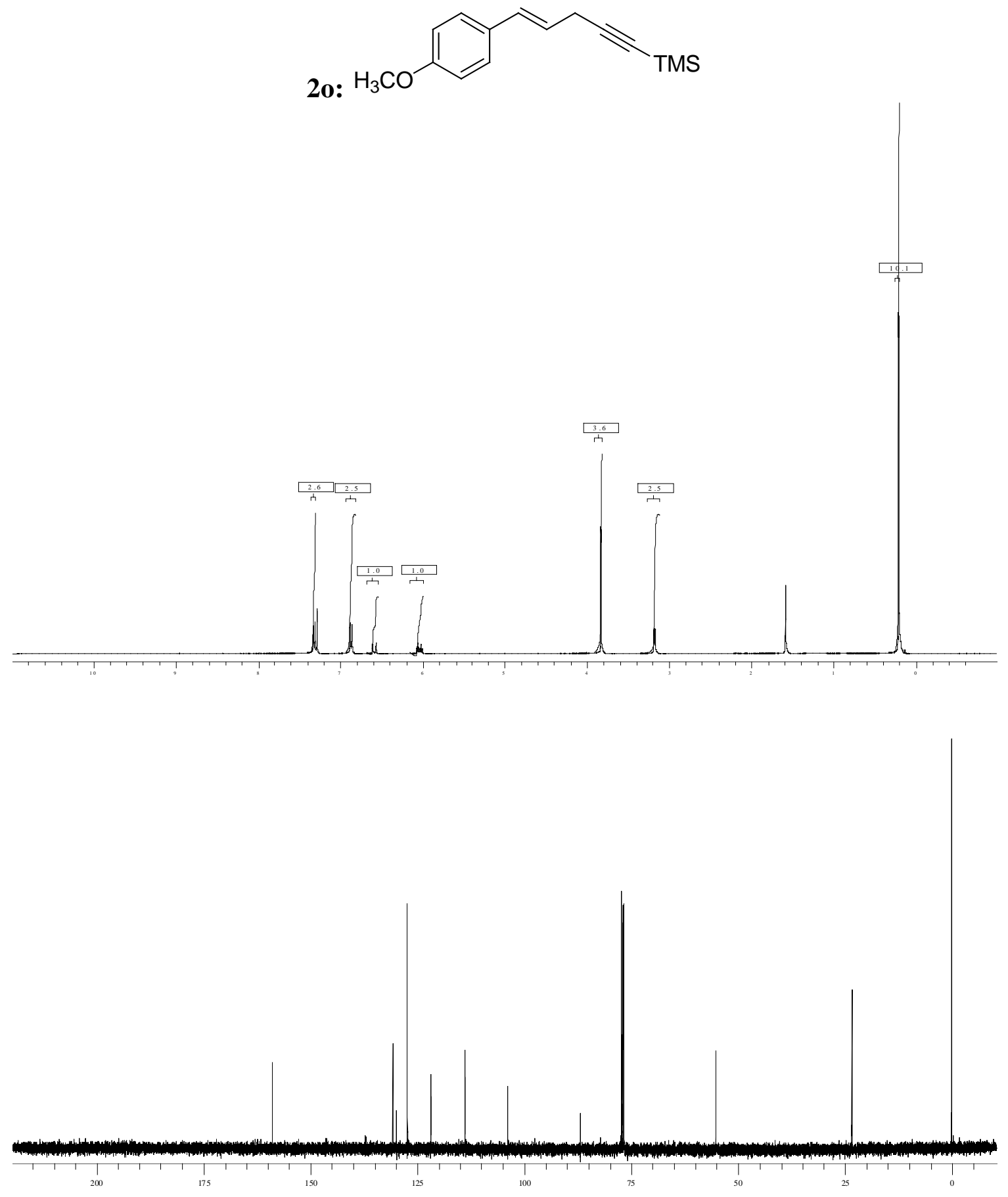

1-[(E)-5-Phenyl-1-penten-4-ynyl]benzene (2o): ${ }^{1} \mathrm{H}$ NMR $\left(400 \mathrm{MHz}, \mathrm{CDCl}_{3}\right): \delta=0.21$ $(\mathrm{s}, 9 \mathrm{H}), 3.17(\mathrm{dd}, J=1.1 \mathrm{~Hz}, J=5.5 \mathrm{~Hz}, 2 \mathrm{H}), 3.83(\mathrm{~s}, 3 \mathrm{H}), 6.03(\mathrm{dt}, J=6.1 \mathrm{~Hz}, J=$ $16.0 \mathrm{~Hz}, 1 \mathrm{H}), 6.56(\mathrm{~d}, J=15.7 \mathrm{~Hz}, 1 \mathrm{H}), 6.87(\mathrm{~d}, J=8.4 \mathrm{~Hz}, 2 \mathrm{H}), 7.32(\mathrm{~d}, J=8.6 \mathrm{~Hz}, 2$ $\mathrm{H}) ;{ }^{13} \mathrm{C}\left\{{ }^{1} \mathrm{H}\right\}$ NMR $\left(100 \mathrm{MHz}, \mathrm{CDCl}_{3}\right): \delta=0.1,23.4,55.2 .86 .8,103.9,113.9,121.7$, 127.3, 129.8, 130.7, 158.9; IR (neat): 759, 840, 966, 1033, 1174, 1247, 1298, 1512, 1608, 2835, 2898, $2956 \mathrm{~cm}^{-1}$; HRMS calcd for $\mathrm{C}_{15} \mathrm{H}_{20} \mathrm{OSi} 244.4042$, found (M+H) 245.1362. 


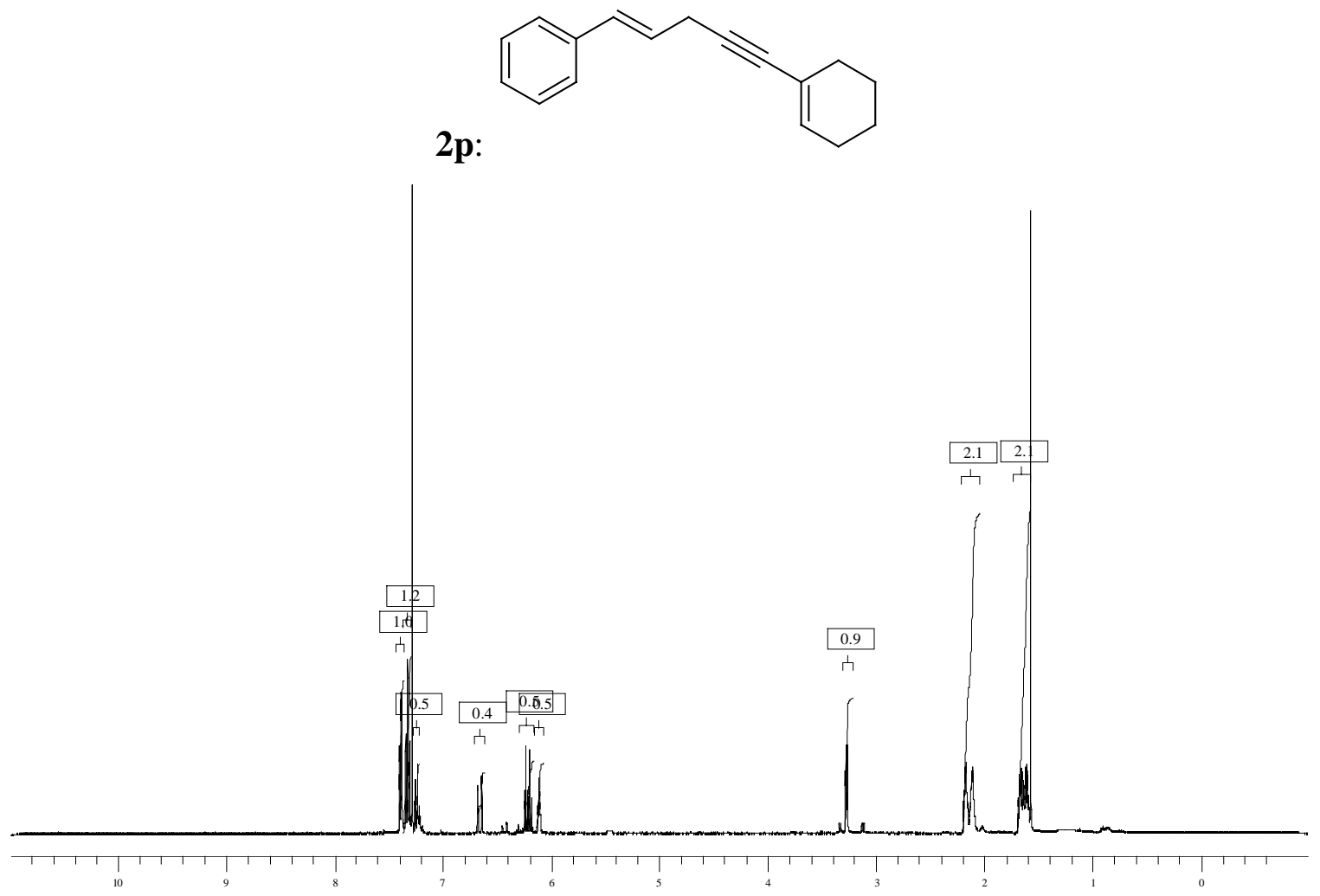

(E)-(5-Cyclohexenylpent-1-en-4-ynyl)benzene (2p): ${ }^{1} \mathrm{H}$ NMR (400 MHz, $\left.\mathrm{CDCl}_{3}\right): \delta=$ 1.59-1.69 (m, 4 H), 2.07-2.13 (m, 2 H), 2.14-2.19 (m, 2 H), 3.26 (d, J = 5.5 Hz, 2 H), 6.09-6.12 (m $1 \mathrm{H}), 6.21(\mathrm{dt}, J=15.7 \mathrm{~Hz}, J=5.8 \mathrm{~Hz}, 1 \mathrm{H}), 6.65(\mathrm{~d}, J=15.7 \mathrm{~Hz}, 1 \mathrm{H})$, $7.24(\mathrm{~d}, J=7.3 \mathrm{~Hz}, 1 \mathrm{H}), 7.32(\mathrm{t}, J=7.3 \mathrm{~Hz}, 2 \mathrm{H}), 7.38(\mathrm{~d}, J=7.0 \mathrm{~Hz}, 2 \mathrm{H}) ;{ }^{13} \mathrm{C}\left\{{ }^{1} \mathrm{H}\right\}$ NMR $\left(100 \mathrm{MHz}, \mathrm{CDCl}_{3}\right): \delta=21.5,22.3,22.9,25.6,29.2,84.5,86.7,120.6,124.7$, 126.2, 127.2, 128.5, 131.1, 133.9, 137.5; IR (neat): 692, 756, 1027, 1172, 1247, 1299, 1434, 1488, 1735, 2927, 2950, $3031 \mathrm{~cm}^{-1}$; HRMS calcd for $\mathrm{C}_{17} \mathrm{H}_{18} 222.1409$, found $(\mathrm{M}+\mathrm{H}) \mathrm{C}_{17} \mathrm{H}_{19} \mathrm{O}_{2} 223.1487$. 


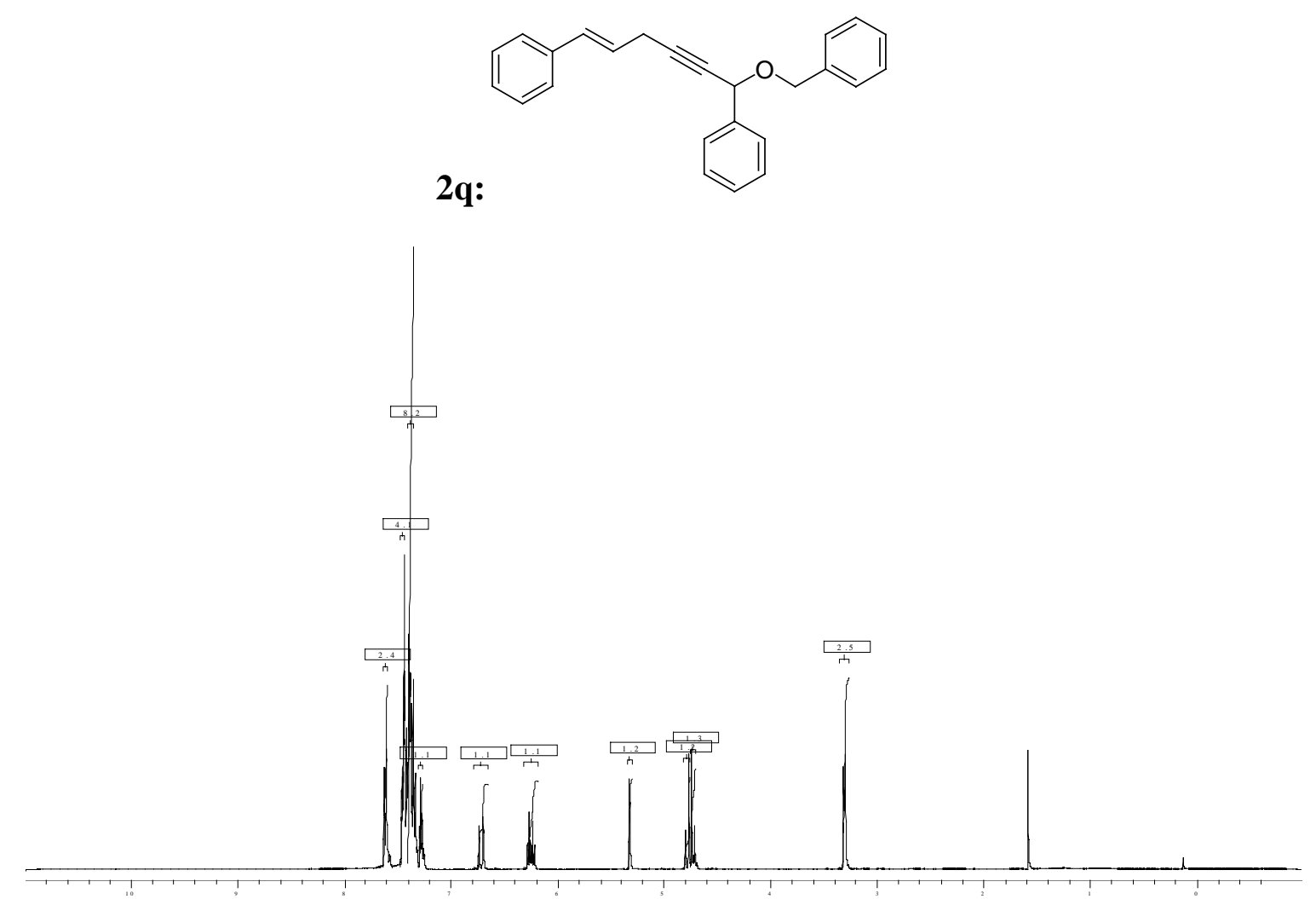

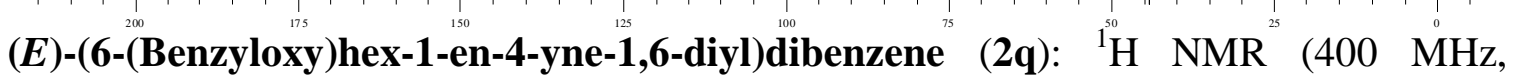
$\left.\mathrm{CDCl}_{3}\right): \delta=3.30(\mathrm{dt}, J=1.7 \mathrm{~Hz}, J=5.5 \mathrm{~Hz}, 2 \mathrm{H}), 4.72(\mathrm{~d}, J=11.6 \mathrm{~Hz}, 1 \mathrm{H}), 4.77(\mathrm{~d}, J=$ $11.6 \mathrm{~Hz}, 1 \mathrm{H}), 5.32(\mathrm{t}, J=1.7 \mathrm{~Hz}, 1 \mathrm{H}), 6.24(\mathrm{dt}, J=5.5 \mathrm{~Hz}, J=15.4 \mathrm{~Hz}, 1 \mathrm{H}), 6.71(\mathrm{dt}, J$ $=1.7 \mathrm{~Hz}, J=15.7 \mathrm{~Hz}, 1 \mathrm{H}), 7.25-7.45(\mathrm{~m}, 13 \mathrm{H}), 7.61(\mathrm{~d}, J=7.6 \mathrm{~Hz}, 2 \mathrm{H}) ;{ }^{13} \mathrm{C}\left\{{ }^{1} \mathrm{H}\right\}$ NMR $\left(100 \mathrm{MHz}, \mathrm{CDCl}_{3}\right): \delta=22.4,69.8,70.6,80.4,85.1,123.9,126.2,127.3,127.4$, 127.6, 128.0, 128.2, 128.3, 128.4, 128.5, 131.4, 136.9, 137.8, 139.0; IR (neat): 696, 730, 964, 1027, 1058, 1083, 1205, 1303, 1452, 1494, 2862, 2921, 3028, $3060 \mathrm{~cm}^{-1}$; HRMS calcd for $\mathrm{C}_{25} \mathrm{H}_{22} \mathrm{O} 338.1671$, found $(\mathrm{M}+\mathrm{H}) 339.1749$. 


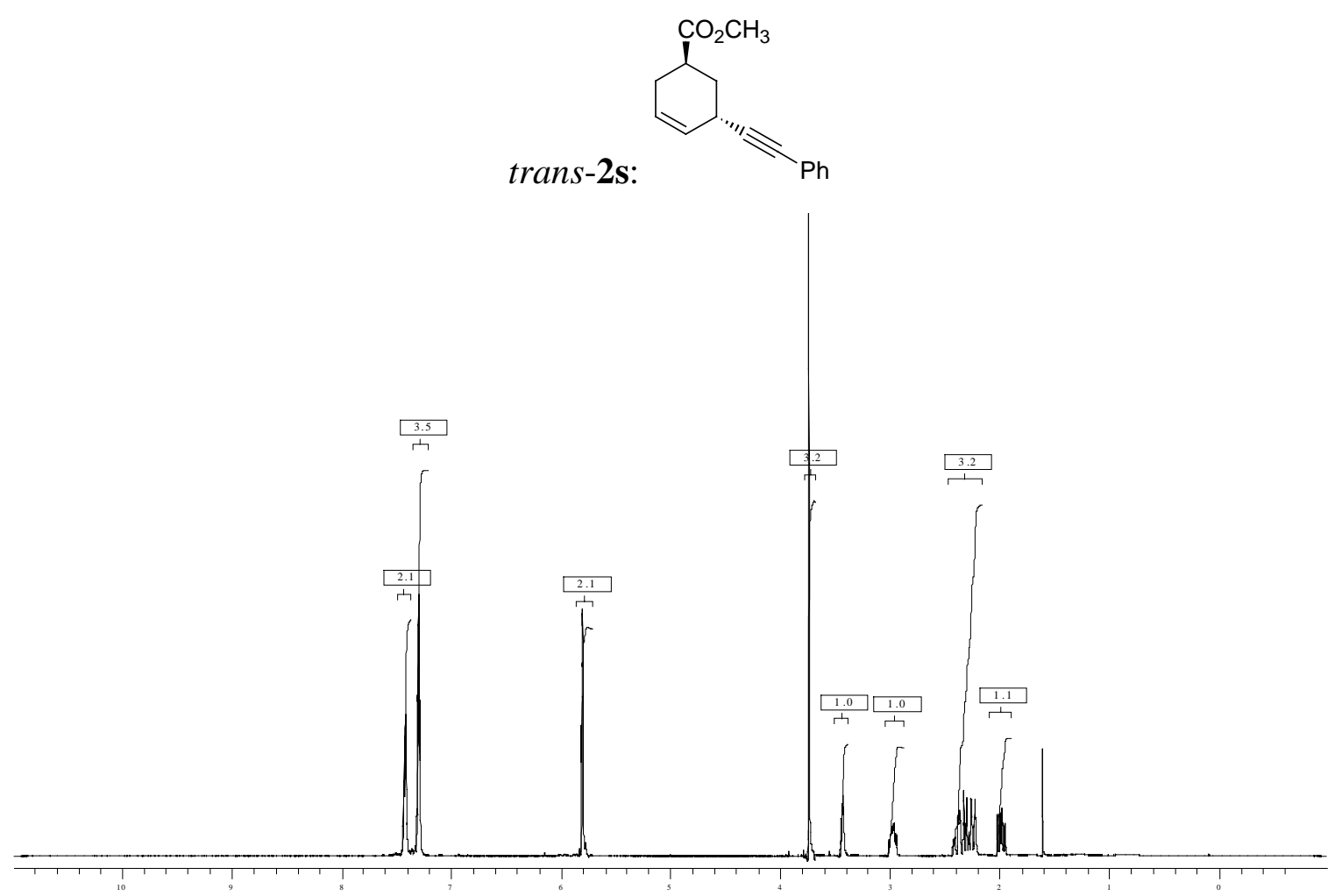

Methy 5-(phenylethynyl)cyclohex-3-enecarboxylate (trans-2s): ${ }^{1} \mathrm{H}$ NMR $(400 \mathrm{MHz}$, $\left.\mathrm{CDCl}_{3}\right): \delta=1.98(\mathrm{ddd}, J=5.5 \mathrm{~Hz}, J=5.5 \mathrm{~Hz}, J=5.5 \mathrm{~Hz}, 1 \mathrm{H}), 2.22-2.42(\mathrm{~m}, 3 \mathrm{H}), 2.94-$ $3.01(\mathrm{~m} 1 \mathrm{H}), 3.43(\mathrm{~d}, \mathrm{dd}, J=3.2 \mathrm{~Hz}, J=5.2 \mathrm{~Hz}, 1 \mathrm{H}), 3.74(\mathrm{~s}, 3 \mathrm{H}), 5.80(\mathrm{~d}, J=2.0 \mathrm{~Hz}$, $2 \mathrm{H}), 7.29(\mathrm{t}, J=6.7 \mathrm{~Hz}, 3 \mathrm{H}), 7.41(\mathrm{~d}, J=6.1 \mathrm{~Hz}, 2 \mathrm{H}) ;{ }^{13} \mathrm{C}\left\{{ }^{1} \mathrm{H}\right\} \mathrm{NMR}(100 \mathrm{MHz}$, $\left.\mathrm{CDCl}_{3}\right): \delta=26.3,27.3,30.8,36.1,51.7,81.5,91.1,123.5,126.1,126.2,127.7,128.1$, 131.6, 175.8; IR (neat): 692, 756, 1027, 1172, 1247, 1299, 1434, 1488, 1735, 2927, 2950, $3031 \mathrm{~cm}^{-1}$; HRMS calcd for $\mathrm{C}_{16} \mathrm{H}_{16} \mathrm{O}_{2} 240.1150$, found $(\mathrm{M}+\mathrm{H}) \mathrm{C}_{16} \mathrm{H}_{17} \mathrm{O}_{2} 241.1229$. 


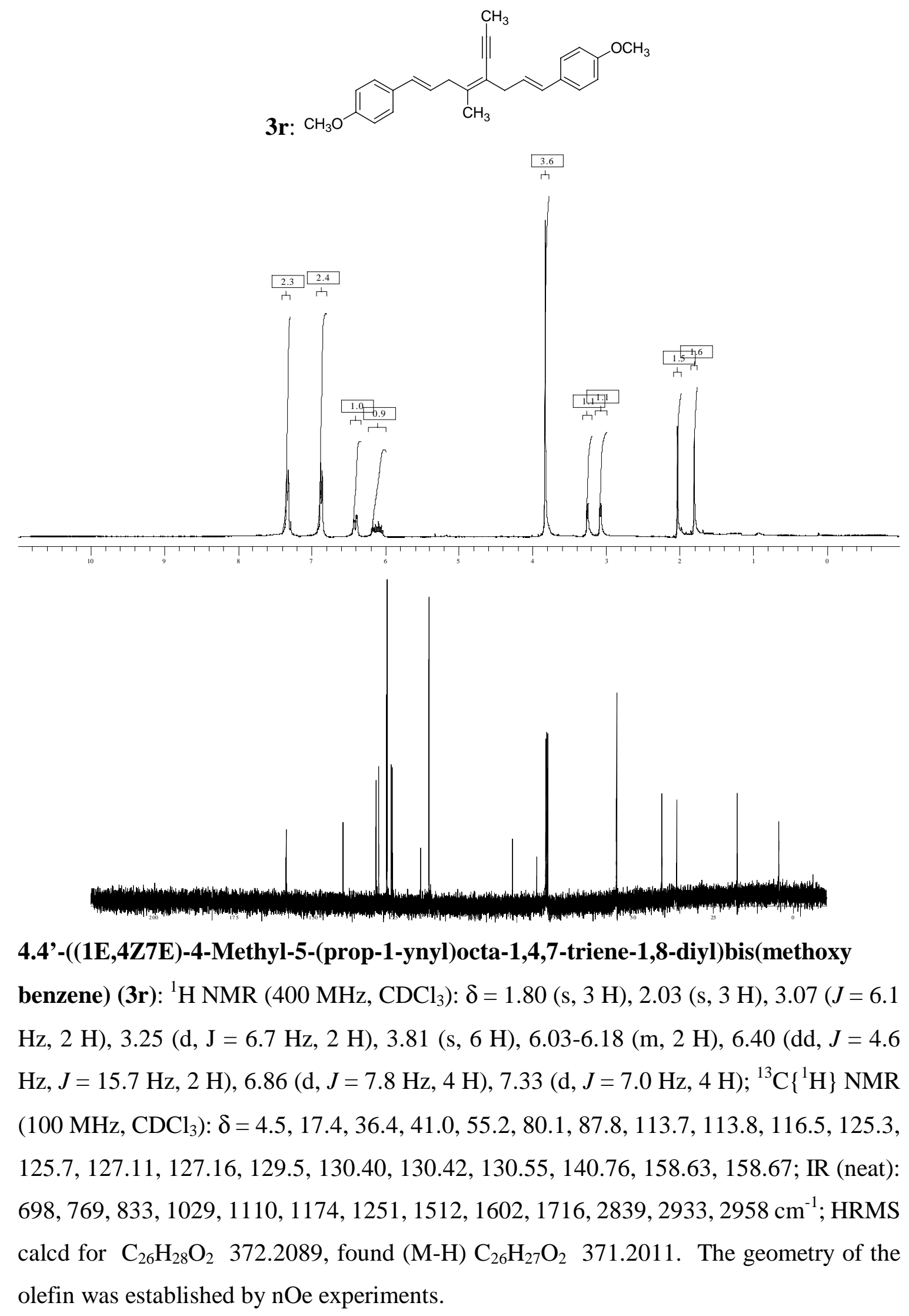


Crossover Experiment Results. Allylic propiolates 1d and 1m and $\mathrm{Pd}\left(\mathrm{PPh}_{3}\right)_{4}(10 \mathrm{~mol}$ $\%$ ) were dissolved in toluene- $d_{8}$ and this reaction mixture was heated to $75{ }^{\circ} \mathrm{C}$. The ${ }^{1} \mathrm{H}$ NMR spectrum shown below was collected after a 45 min. reaction period. Extensive crossover is evident from the positive identification of the four possible allylic propiolates.
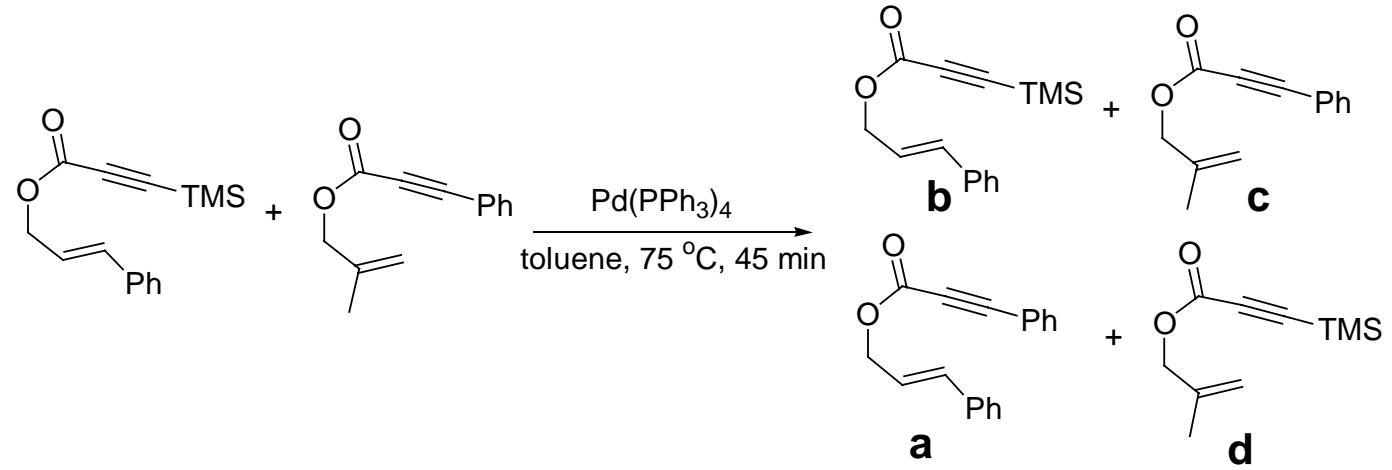

${ }^{1} \mathrm{H}$ Spectrum depicting the $\mathrm{OCH}_{2}$ of the following compounds

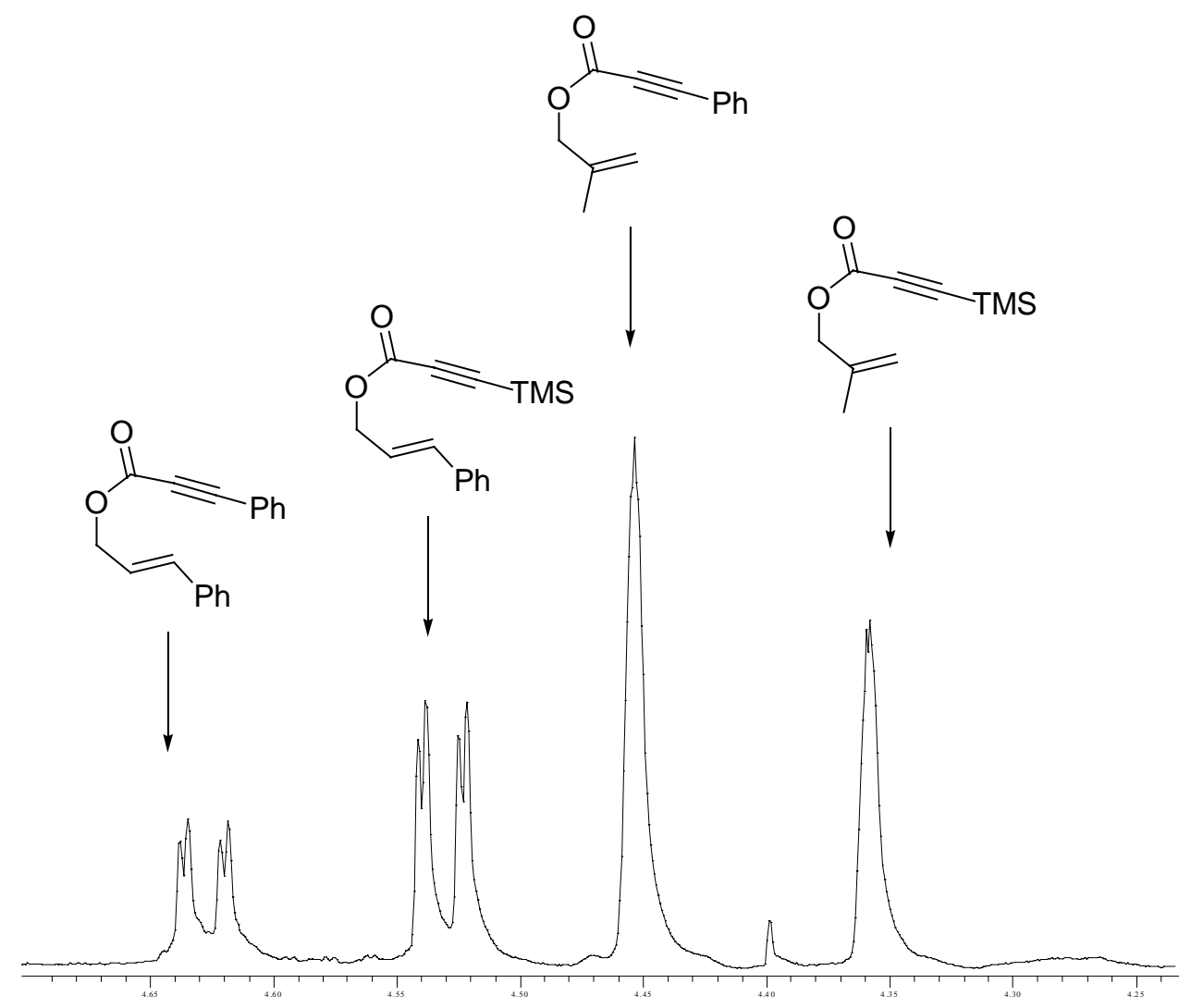




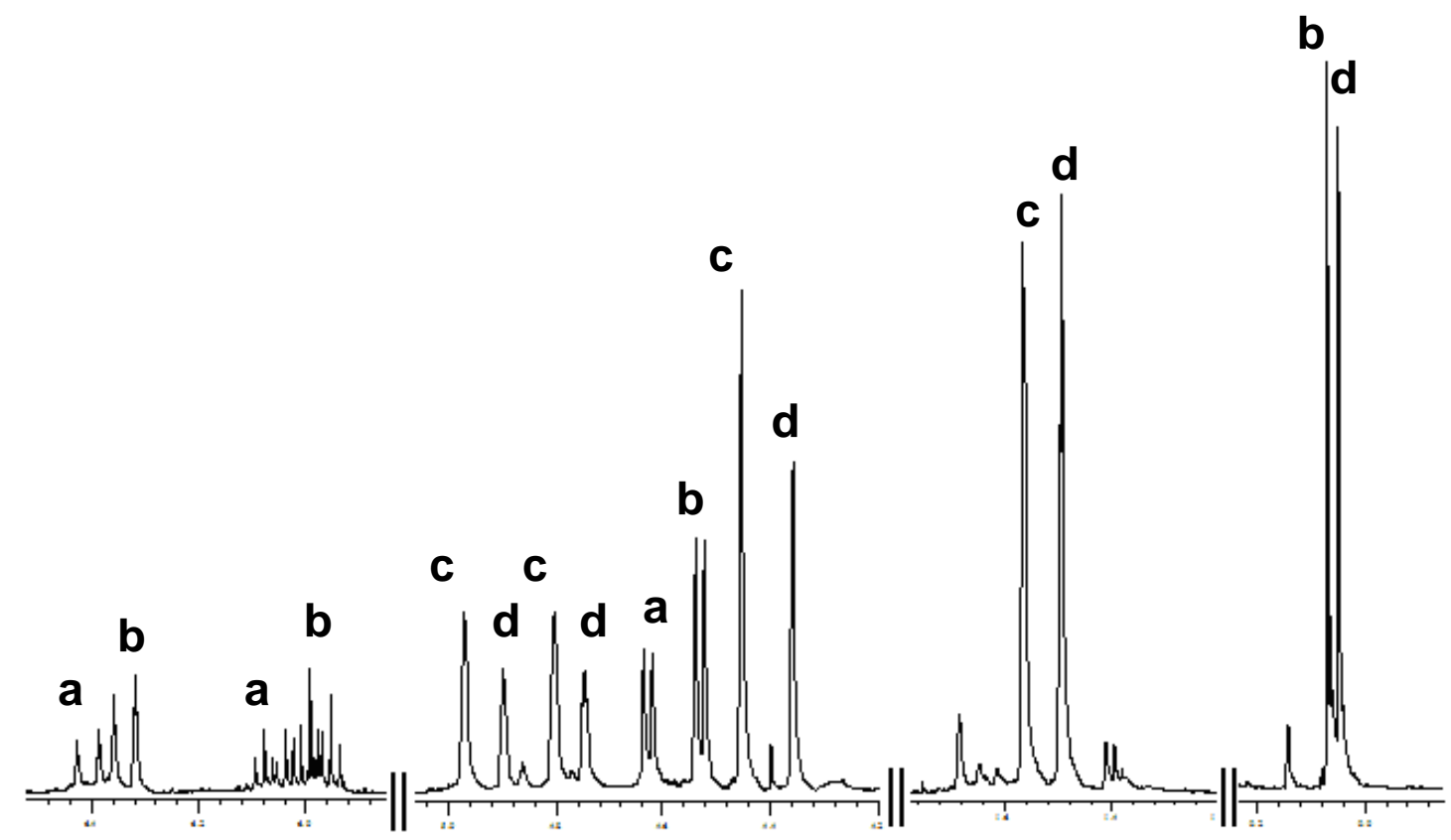

${ }^{1}$ (a) Klein, J.; Brenner, S. Tetrahedron 1970, 26, 5807-19. (b) Cui, D.-M.; Hashimoto, N.; Ikeda, S.-i.; Sato, Y. 1995, 60, 5752-6.

${ }^{2}$ Chen, H.; Deng, M.-Z. J. Organomet. Chem. 2000, 603, 189-193.

${ }^{3}$ Nishikawa, T.; Isobe, M. Biosci., Biotechnol., Biochem. 1999, 63, 238-242. 